\title{
A new alpha power transformed family of distributions: properties and applications to the Weibull model
}

\author{
I. Elbatal ${ }^{\mathrm{a}}$, Zubair Ahmad ${ }^{\mathrm{b}}$, M. Elgarhy ${ }^{\mathrm{c}, *}$, Abdullah M. Almarashid $^{\mathrm{d}}$ \\ ${ }^{a}$ Department of Mathematics and Statistics, College of Science, Al Imam Mohammad Ibn Saud Islamic University (IMSIU), Saudi Arabia. \\ ${ }^{b}$ Department of Statistics, Quaid-i-Azam University 45320, Islamabad 44000, Pakistan. \\ ${ }^{c}$ Vice Presidency for Graduate Studies and Scientific Research, University of Jeddah, Jeddah, KSA. \\ ${ }^{d}$ Statistics Department, Faculty of Science, King AbdulAziz University, Jeddah, Kingdom of Saudi Arabia.
}

\begin{abstract}
In this article, a new technique of alpha-power transformation is used to propose a new class of lifetime distributions. Four special models of the new family are presented. Some mathematical properties of the proposed model including estimation of the unknown parameters using the method of maximum likelihood are discussed. For the illustrative purposes of the new proposal, a three-parameter special model of this class, namely, new alpha-power transformed Weibull distribution is considered in detail. The proposed distribution offers greater distributional flexibility and is able to model data with increasing, decreasing, and constant or more importantly with bathtub-shaped failure rates. Type- 1 and Type-II censoring estimation are discussed. A simulation study based on complete sample of the new model is also carried out. Finally, the usefulness and efficiency of the new proposal is illustrated by analyzing two real data sets.
\end{abstract}

Keywords: Alpha- power transformation, Weibull distribution, type-I and type-II censoring, bathtub shape, moment generating function, maximum likelihood estimation.

2010 MSC: 60E05, 62E10, 62N05.

(C)2019 All rights reserved.

\section{Introduction}

In the practice of distribution theory, the development of new statistical models is a prominent research topic. The literature is filled with such distributions that are very worthwhile in predicting and modeling real world phenomena. A number of classical distributions have been used comprehensively over the past decades for modeling data in several applied areas including bio-medical analysis, reliability engineering, economics, forecasting, astronomy, demography and insurance. After the massive work by Pearson [23] for proposing new statistical distributions using the system of differential equation method, numerous general procedures have been suggested for introducing new family of distributions. Another prominent

\footnotetext{
*Corresponding author

Email addresses: iielbatal@imamu.edu.sa (I. Elbatal), z.ferry21@gmail.com (Zubair Ahmad), m_elgarhy85@yahoo.com (M.

Elgarhy), aalmarashi@kau.edu.sa (Abdullah M. Almarashi)
}

doi: $10.22436 /$ jnsa.012.01.01

Received: 2018-07-05 Revised: 2018-08-04 Accepted: 2018-08-15 
method generated on differential equation was introduced by Burr [4] which can take on an extensive variety of shapes of the continuous statistical distributions. Since 1980, methodologies of proposing new distributions moved to the introduction of additional parameters to an existing class of distributions to increase the level of flexibility. For instance, Mudholkar and Srivastava [19] and Marshall and Olkin [18] proposed new method of adding parameters to existing distributions. Some of the well-known family of distributions are beta-generated by Eugene et al. [10], Weibull-G studied by Bourguignon et al. [3], Garhy-G of Elgarhy et al. [9], Kwmaraswamy (Kw-G) introduced by Cordeiro and de Castero [5], Type II half logistic-G of Hassan et al. [14], exponentiated extended-G proposed by Elgarhy et al. [8], the Kumaraswamy Weibull by Hassan and Elgarhy [13], exponentiated Weibull by Hassan and Elgarhy [12], Odd Frechet-G by Haq and Elgarhy [11], and Muth-G by Almarashi and Elgarhy [2]. For a brief review, one may refer to Kotz and Vicari [15] which reviewed the primary development of statistical distributions. Recently, Mahdavi and Kundu [17] introduced a new method for generating lifetime distributions named as alpha-power transformation (APT) method, defined by the cumulative distribution function (cdf) is given below

$$
G(x)= \begin{cases}\frac{\alpha^{F(x)}-1}{\alpha-1}, & \text { if } \alpha>0, \alpha \neq 1, x \in R, \\ F(x), & \text { if } \alpha=1,\end{cases}
$$

the quantity $F(x)$ in (1.1) is a cdf of the baseline distribution. Using the cdf of exponential distribution in (1.1) Mahdavi and Kundu [17] introduced a two-parameter model named as alpha-power exponential (APE) distribution. The cdf of the APE distribution is given by

$$
G(x)= \begin{cases}\frac{\alpha^{\left(1-e^{-\gamma x}\right)}-1}{\alpha-1}, & \text { if } \alpha, \gamma>0, \alpha \neq 1, x>0, \\ 1-e^{-\gamma x}, & \text { if } \alpha=1 .\end{cases}
$$

Using (1.1), Dey et al. [7] introduced the alpha-power transformed Weibull (APTW) distribution which has the cdf given as follows

$$
G(x)= \begin{cases}\frac{\alpha^{\left(1-e^{-\gamma x^{\theta}}\right)}-1}{\alpha-1}, & \text { if } \alpha, \gamma, \theta>0, \alpha \neq 1, x>0, \\ 1-e^{-\gamma x^{\theta}}, & \text { if } \alpha=1 .\end{cases}
$$

Several lifetime models such as Weibull, alpha-power transformed exponential (APTE), and alpha-power transformed Rayleigh (APTR) distributions can be obtained as special models of the APTW distribution. In the present article, we use a new scheme to add an additional parameter to introduce a new class of distributions. The new proposal may be named as new alpha-power transformation (NAPT) method. The cdf of the proposed family is defined by the following expression

$$
G(x ; \Theta)= \begin{cases}\frac{F(x ; \xi) \alpha^{F(x ; \xi)},}{\alpha}, & \text { if } \alpha, \xi>0, \quad \alpha \neq 1, \quad X \in R, \\ F(x ; \xi), & \text { if } \alpha=1,\end{cases}
$$

where $\Theta=(\alpha, \xi)$. From the expression in (1.2), it is well clear that if $\alpha \neq 1$, thenG $(x ; \Theta)$ is a weighted version of $F(x ; \xi)$, where the weight is $w(x)=\alpha^{F(x ; \xi)} / \alpha$. So, the expression in (1.2) can be written as

$$
\mathrm{G}(x ; \Theta)=\mathrm{F}(x ; \xi) w(x) .
$$

Weighted distributions play an essential role in the practice of statistical distribution theory, for detail see Patil and Ord [21] and Patil and Rao [22]. The probability density function (pdf) corresponding to (1.2) is given by

$$
g(x ; \Theta)=\frac{f(x ; \xi) \alpha^{F(x ; \xi)}}{\alpha}[1+\log (\alpha) F(x ; \xi)], \quad \text { if } \alpha>0, \quad \alpha \neq 1 .
$$


The corresponding survival function (sf), hazard rate function (hrf), reversed hazard rate function (rhrf), and cumulative hazard rate function (chrf) are provided in (1.4)-(1.7), respectively.

$$
\begin{aligned}
& S(x ; \Theta)=\frac{\alpha-F(x ; \xi) \alpha^{F(x ; \xi)}}{\alpha}, \text { if } \alpha>0, \alpha \neq 1 . \\
& h(x ; \Theta)=\frac{f(x ; \xi) \alpha^{F(x ; \xi)}[1+\log (\alpha) F(x ; \xi)]}{\alpha-F(x ; \xi) \alpha^{F(x ; \xi)}}, \text { if } \alpha>0, \alpha \neq 1 . \\
& r(x ; \Theta)=\frac{f(x ; \xi)[1+\log (\alpha) F(x ; \xi)]}{F(x ; \xi)}, \text { if } \alpha>0, \alpha \neq 1 . \\
& H(x ; \Theta)=-\log \left(\frac{\alpha-F(x ; \xi) \alpha^{F(x ; \xi)}}{\alpha}\right), \text { if } \alpha>0, \alpha \neq 1 .
\end{aligned}
$$

Henceforth, a random variable $X$ with pdf given by (1.3) will be denoted by $X \sim \operatorname{NAPT}(x ; \Theta)$. Furthermore, the key motivations for using the NAPT family in practice are as:

1. A very simple and efficient method of inducting an additional parameter to generalize the existing distributions.

2. To improve the characteristics and flexibility of the existing distributions.

3. To introduce the extended version of the baseline distribution having closed form of cdf, sf as well as hrf.

4. To extend the existing distributions by introducing only one parameter, rather than adding two or more parameters.

5. To define special models capable of modeling with monotonic and non-monotonic hazard functions.

6. To provide better fits than other modified models having same or higher number of parameters.

We are motivated to study a special model of this class named as new alpha-power transformed Weibull (NAPTW) distribution. The suggested model offers greater distributional flexibility and is able to model lifetime with monotonically increasing, decreasing and constant or more importantly with bathtub-shaped failure rates. The rest of the article is structured as follows. In Section 2, we provide a mixture representation and importance of the new proposal. Section 3, provides the limiting behavior of the new family. Basic mathematical properties including quantile function, ordinary and incomplete moments, moment generating function and probability weighted moments are derived in Section 4 . Section 5 considers four special models of the proposed family and discusses its special cases. Section 6 considers the maximum likelihood estimation and simulation. Censoring estimation is discussed in Section 7. Two real data sets are analyzed in Section 8. Finally, Section 9 concludes the article.

\section{Useful expansions and importance of NAPT family}

This section presents the importance and mixture representation for the pdf and cdf of the proposed family. Using the series representation in the form

$$
\alpha^{v}=\sum_{i=0}^{\infty} \frac{(\log \alpha)^{i}}{i !} v^{i}
$$

so, the pdf in (1.3) can be written as

$$
g(x ; \Theta)=\sum_{i=0}^{\infty} \eta_{i} f(x ; \xi)[F(x ; \xi)]^{i}+\sum_{i=0}^{\infty} \eta_{i}^{\prime} f(x ; \xi)[F(x ; \xi)]^{i+1},
$$

where, $\eta_{i}=\frac{1}{\alpha} \frac{(\log \alpha)^{i}}{i !}$, and $\eta_{i}^{\prime}=\frac{1}{\alpha} \frac{(\log \alpha)^{i+1}}{i !}$. 
Furthermore, another form of the pdf in (2.1), which provides the following infinite combination

$$
g(x ; \Theta)=\sum_{i=0}^{\infty} \frac{\eta_{i}}{i+1} a f(x ; \xi)[F(x ; \xi)]^{a-1}+\sum_{i=0}^{\infty} \frac{\eta_{i}^{\prime}}{i+2} b f(x ; \xi)[F(x ; \xi)]^{b-1},
$$

where, $a=i+1$, and $b=i+2$.

We can also write (2.2) as

$$
g(x ; \Theta)=\sum_{i=0}^{\infty} \frac{\eta_{i}}{i+1} h_{i+1}(x ; \xi)+\sum_{i=0}^{\infty} \frac{\eta_{i}^{\prime}}{i+2} h_{i+2}(x ; \xi),
$$

where, $h_{i+1}(x ; \xi)=a f(x ; \xi)[F(x ; \xi)]^{a-1}$, and $h_{i+2}(x ; \xi)=b f(x ; \xi)[F(x ; \xi)]^{b-1}$, are the exponentiated generated (Exp-G) densities with power parameters $a$ and $b$, respectively. From the density derived in (2.3), it is well clear that the proposed family can be expressed as an infinite combinations of exponentiated family of distributions. Henceforth, the NAPT family of distributions can also be used in modeling data, where data modeling using compound (mixture) distributions are required.

The cdf of the NAPT family can be written as

$$
\mathrm{G}(x ; \Theta)=\sum_{i=0}^{\infty} \frac{(\log \alpha)^{i}}{i ! \alpha} \mathrm{F}(x ; \xi)^{i+1} .
$$

Let $u$ is an integer, then the expression for $g(x ; \Theta)[G(x ; \Theta)]^{u}$ is derived as

$$
g(x ; \Theta)[G(x ; \Theta)]^{u}=\sum_{i=0}^{\infty} K_{i} f(x ; \xi)[F(x ; \xi)]^{\mathfrak{u}+\mathfrak{i}}+\sum_{i=0}^{\infty} K_{i}^{\prime} f(x ; \xi)[F(x ; \xi)]^{u+i+1},
$$

where, $K_{i}=\frac{1}{\alpha^{u+1}} \frac{(\log \alpha)^{i}(u+1)^{i}}{i !}$, and $K_{i}^{\prime}=\frac{1}{\alpha^{u+1}} \frac{(\log \alpha)^{i+2}(u+1)^{i}}{i !}$.

The density function in (2.2) and the other function derived in (2.4) can be used quite effectively to derive numerous mathematical properties of the NAPT family. For instance, we can obtain closed form solution for the ordinary and probability weighted moments of any special case of the NAPT family.

\section{Limiting behavior of the NAPT family}

In this section, we study the behavior of the cdf, pdf, sf, hrf, and rhrf of the NAPT family of distributions as $x \rightarrow-\infty$ and $x \rightarrow \infty$.

Proposition 3.1. The limiting behavior of (1.2), (1.3), (1.4), (1.5), and (1.6) as $x \rightarrow-\infty$ are given by

$$
\begin{aligned}
& \mathrm{G}(x ; \Theta) \rightarrow 0 \text { as } x \rightarrow-\infty, \\
& \mathrm{g}(x ; \Theta) \rightarrow \frac{f(x ; \xi)}{\alpha} \text { as } x \rightarrow-\infty, \\
& \mathrm{S}(x ; \Theta) \rightarrow 1 \text { as } x \rightarrow-\infty, \\
& \mathrm{h}(x ; \Theta) \rightarrow \frac{f(x ; \xi)}{\alpha} \text { as } x \rightarrow-\infty, \\
& \mathrm{r}(x ; \Theta) \rightarrow \infty \text { as } x \rightarrow-\infty .
\end{aligned}
$$

Proposition 3.2. The limiting behavior of (1.2), (1.3), (1.4), (1.5), and (1.6) as $x \rightarrow \infty$ are given by

$$
\begin{aligned}
\mathrm{G}(x ; \Theta) & \rightarrow 1 \text { as } x \rightarrow \infty, \\
\mathrm{g}(x ; \Theta) & \rightarrow \mathrm{f}(\mathrm{x} ; \xi)[1+(\log \alpha)] \text { as } x \rightarrow \infty, \\
\mathrm{S}(\mathrm{x} ; \Theta) & \rightarrow 0 \text { as } x \rightarrow \infty, \\
\mathrm{h}(\mathrm{x} ; \Theta) & \rightarrow \infty \text { as } x \rightarrow \infty, \\
\mathrm{r}(\mathrm{x} ; \Theta) & \rightarrow \mathrm{f}(\mathrm{x} ; \xi)[1+(\log \alpha)] \text { as } x \rightarrow \infty .
\end{aligned}
$$




\section{Basic mathematical properties}

This section considers some key mathematical properties of the proposed family.

\subsection{Ordinary moments}

Speaking broadly, we always need to keep in mind the importance of moments in any statistical analysis particularly in applied fields. For example, through moments the important characteristics such as tendency, dispersion, skewness, and kurtosis of a distribution can be studied.

Let $X \sim \operatorname{NAPT}(x ; \Theta)$, then the $r^{\text {th }}$ ordinary moment of $X$ is derived as follows

$$
\begin{aligned}
\mu_{r}^{\prime} & =\int_{-\infty}^{\infty} \sum_{i=0}^{\infty} x^{r} \eta_{i} f(x ; \xi)[F(x ; \xi)]^{i} d x+\int_{-\infty}^{\infty} \sum_{i=0}^{\infty} x^{r} \eta_{i}^{\prime} f(x ; \xi)[F(x ; \xi)]^{i+1}, \\
\mu_{r}^{\prime} & =\sum_{i=0}^{\infty} \eta_{i} v_{r, i}+\sum_{i=0}^{\infty} \eta_{i}^{\prime} v_{r, i+1},
\end{aligned}
$$

where, $v_{r, i}=\int_{-\infty}^{\infty} x^{r} f(x ; \xi)[F(x ; \xi)]^{i} d x$, and $v_{r, i+1}=\int_{-\infty}^{\infty} x^{r} f(x ; \xi)[F(x ; \xi)]^{i+1} d x$.

\subsection{Quantile function}

The quantile function of NAPT random variable $X$ is given by

$$
\log (F(x ; \xi))+F(x ; \xi) \log (\alpha)-\log (\alpha \mathrm{U})=0 .
$$

Using the previous equation, we can generate random sample from NAPT family by using $\mathrm{U}$ as uniform random number.

\subsection{Incomplete moments}

The incomplete moment is one of the most usual approaches used to determine the moments of a probability distribution. These moments play an essential role in determining the Bonferroni and Lorenz curves. The $r^{\text {th }}$ incomplete moment of $X$ is derived as

$$
\begin{aligned}
& \Upsilon_{r}(y)=\int_{-\infty}^{y} \sum_{i=0}^{\infty} x^{r} \eta_{i} f(x ; \xi)[F(x ; \xi)]^{i} d x+\int_{-\infty}^{y} \sum_{i=0}^{\infty} x^{r} \eta_{i}^{\prime} f(x ; \xi)[F(x ; \xi)]^{i+1}, \\
& \Upsilon_{r}(y)=\sum_{i=0}^{\infty} \eta_{i} b_{r, i}+\sum_{i=0}^{\infty} \eta_{i}^{\prime} b_{r, i+1}
\end{aligned}
$$

where, $b_{r, i}=\int_{-\infty}^{y} x^{r} f(x ; \xi)[F(x ; \xi)]^{i} d x$, and $b_{r, i+1}=\int_{-\infty}^{y} x^{r} f(x ; \xi)[F(x ; \xi)]^{i+1} d x$.

Furthermore, the conditional measures which play an important role in predictions can be derived as

$$
\begin{aligned}
& E\left(X^{r} / X>t\right)=\frac{1}{S(t ; \xi)}\left(\int_{t}^{\infty} \sum_{i=0}^{\infty} x^{r} \eta_{i} f(x ; \xi)[F(x ; \xi)]^{i} d x+\int_{t}^{\infty} \sum_{i=0}^{\infty} x^{r} \eta_{i}^{\prime} f(x ; \xi)[F(x ; \xi)]^{i+1}\right), \\
& E\left(X^{r} / X>t\right)=\frac{1}{S(t ; \xi)}\left(\sum_{i=0}^{\infty} \eta_{i} \Lambda_{r, i}+\sum_{i=0}^{\infty} \eta_{i}^{\prime} \Lambda_{r, i+1}\right),
\end{aligned}
$$

where $\Lambda_{r, i}=\int_{t}^{\infty} x^{r} f(x ; \xi)[F(x ; \xi)]^{i} d x$, and $\Lambda_{r, i+1}=\int_{t}^{\infty} x^{r} f(x ; \xi)[F(x ; \xi)]^{i+1} d x$.

\subsection{The probability weighted moments}

The probability weighted moments (pwms) is another approach that is used to obtain the moments of statistical distributions whose inverse form cannot be expressed explicitly. For a random variable $\mathrm{X}$ with 
pdf given by (2.4), the pwms represented by $\tau_{r, u}$ is derived as

$$
\tau_{r, u}=E\left[X^{r} G(x ; \Theta)^{u}\right]=\int_{-\infty}^{\infty} x^{r} G(x ; \Theta)^{u} g(x ; \Theta) d x .
$$

Inserting (2.4) in the previous equation, the pwms of NAPT family is as follows

$$
\begin{aligned}
\tau_{r, u} & =\int_{-\infty}^{\infty} \sum_{i=0}^{\infty} x^{r} K_{i} f(x ; \xi)[F(x ; \xi)]^{u+i} d x+\int_{-\infty}^{\infty} \sum_{i=0}^{\infty} x^{r} K_{i}^{\prime} f(x ; \xi)[F(x ; \xi)]^{u+i+1} d x, \\
\tau_{r, u} & =\sum_{i=0}^{\infty} K_{i} v_{r, u+i}+\sum_{i=0}^{\infty} K_{i}^{\prime} v_{r, u+i+1}
\end{aligned}
$$

where, $v_{r, u+i}=\int_{-\infty}^{\infty} x^{r} f(x ; \xi)[F(x ; \xi)]^{u+i} d x$, and $v_{r, u+i+1}=\int_{-\infty}^{\infty} x^{r} f(x ; \xi)[F(x ; \xi)]^{u+i+1} d x$.

\subsection{Moment generating function}

The moment generating function (mgf) is another most prominent approach to study the behavior of probability distributions. A general expression for the NAPT random variable $X$ is obtained as

$$
M_{x}(t)=\int_{-\infty}^{\infty} e^{t x} g(x ; \Theta) d x=\sum_{r=0}^{\infty} \frac{t^{r}}{r !} \mu_{r}^{\prime}, \quad M_{x}(t)=\sum_{i, r=0}^{\infty} \frac{t^{r}}{r !}\left(\eta_{i} v_{r, i}+\eta_{i}^{\prime} v_{r, i+1}\right) .
$$

\subsection{Residual and mean residual life}

The residual life and reversed residual life associated with a lifetime random variable are of interest in numerous areas of applied sciences such as survival analysis, biometry, actuarial studies and risk management. The residual lifetime of $X$ denoted by $R_{(t)}$ is derived as

$$
R_{(t)}(x)=\frac{S(x+t)}{S(t)}, \quad R_{(t)}(x)=\frac{\alpha-F(x+t ; \xi) \alpha^{F(x+t ; \xi)}}{\alpha-F(t ; \xi) \alpha^{F(t ; \xi)}}
$$

Additionally, the reverse residual life of $X$ denoted by $\bar{R}_{(t)}$ can be derived as

$$
\bar{R}_{(t)}=\frac{S(x-t)}{S(t)}, \quad \bar{R}_{(t)}=\frac{\alpha-F(x-t ; \xi) \alpha^{F(x-t ; \xi)}}{\alpha-F(t ; \xi) \alpha^{F(t ; \xi)}} .
$$

\subsection{Rényi and q-entropy}

Entropy is a statistical tool that is used to measure the variation or uncertainty of a random variable. Let $X$ follow NAPT family with density function given in (1.3), then the Rényi entropy of $X$ is derived as

$$
\mathrm{I}_{\mathrm{R}}(\delta)=\frac{1}{1-\delta} \log [\mathrm{I}(\delta)]
$$

where

$$
\mathrm{I}(\delta)=\int_{\mathrm{R}}[\mathrm{g}(\mathrm{x} ; \Theta)]^{\delta} \mathrm{dx}, \quad \delta>0 \text { and } \delta \neq 1 .
$$

Using the generalized binomial series in the following form

$$
(1+z)^{\delta}=\sum_{i=0}^{\infty}(-1)^{i}\left(\begin{array}{c}
\delta+i-1 \\
i
\end{array}\right) z^{i}
$$

so, we have

$$
[g(x ; \Theta)]^{\delta}=\sum_{i, j=0}^{\infty} \varepsilon_{i, j}[f(x ; \xi)]^{\delta}[F(x ; \xi)]^{i+j}
$$


where,

$$
\varepsilon_{i, j}=\frac{(-1)^{i}(\log \alpha)^{i+j}\left(\begin{array}{c}
\delta+i-1 \\
i
\end{array}\right) \delta^{j}}{j ! \alpha^{\delta}}
$$

Using (4.2) in (4.1), we have

$$
I_{R}(\delta)=\frac{1}{1-\delta} \log \int_{R} \sum_{i, j=0}^{\infty} \varepsilon_{i, j}[f(x ; \xi)]^{\delta}[F(x ; \xi)]^{i+j} d x .
$$

The expression in (4.3) can be used to derive the Rényi entropy for any special model of the NAPT family. Furthermore, the q-entropy, say $\mathrm{H}_{\mathrm{q}}(\mathrm{f})$ is defined by

$$
H_{q}(f)=\frac{1}{1-q} \log \left[1-I_{q}(f)\right],
$$

where $\mathrm{I}(\mathrm{q})=\int_{\mathrm{R}}[\mathrm{g}(\mathrm{x} ; \Theta)]^{\mathrm{q}} \mathrm{d} x, \mathrm{q}>0$ and $\mathrm{q} \neq 1$. From (4.3) one can easily obtain

$$
H_{q}(f)=\frac{1}{1-q} \log \left[1-\int_{R} \sum_{i, j=0}^{\infty} \varepsilon_{i, j}[f(x ; \xi)]^{q}[F(x ; \xi)]^{i+j} d x\right] .
$$

\subsection{Order statistics}

Let $X_{1: k}, X_{2: k}, \ldots, X_{k: k}$ be the order statistics of a random sample $X_{1}, X_{2}, \ldots, X_{k}$ observed from NAPT family with $G(x ; \Theta)$ and $g(x ; \Theta)$, then the density function of $X_{r: k}$ is given by

$$
g_{r: k}(x ; \Theta)=\frac{f(x ; \xi)}{B(r, k-r+1)} \sum_{\nu=0}^{k-r}\left(\begin{array}{c}
k-r \\
v
\end{array}\right)(-1)^{v}[F(x ; \xi)]^{k+v-1},
$$

where, B (.,.)denotes the beta function. The pdf of the $r^{\text {th }}$ order statistic of NAPT family is derived by using (2.4) in (4.4), and replacing $u$ with $k+v-1$, we have

$$
g_{r: k}(x ; \Theta)=\frac{\sum_{i, v=0}^{k-r}\left(\begin{array}{c}
k-r \\
v
\end{array}\right)(-1)^{v}}{B(r, k-r+1)}\left[\psi_{i, v} f(x ; \xi)[F(x ; \xi)]^{k+v+i-1}+\psi_{i, v}^{\prime} f(x ; \xi)[F(x ; \xi)]^{k+v+i}\right],
$$

where, $\psi_{i, v}=\frac{1}{\alpha^{k+v}} \frac{(\log \alpha)^{i}(k+v)^{i}}{i !}$, and $\psi_{i, v}^{\prime}=\frac{1}{\alpha^{k+v}} \frac{(\log \alpha)^{i+2}(k+v)^{i}}{i !}$.

Furthermore, $\mathrm{n}^{\text {th }}$ ordinary moment of the $\mathrm{r}^{\text {th }}$ order statistics for NAPT family is derived as

$$
E\left(X_{r: k}^{n}\right)=\int_{-\infty}^{\infty} X_{r: k}^{n} g_{r: k}(x ; \Theta) d x
$$

using (4.5) in (4.6), we get

$$
\begin{aligned}
E\left(X_{r: k}^{n}\right)= & \frac{\sum_{i, v=0}^{n-r}\left(\begin{array}{c}
k-r \\
v
\end{array}\right)(-1)^{v}}{B(r, k-r+1)} \\
& \times \int_{-\infty}^{\infty}\left[x_{r: k}^{n} \psi_{i, v} f(x ; \xi)[F(x ; \xi)]^{k+v+i-1}+x_{r: k}^{n} \psi_{i, v}^{\prime} f(x ; \xi)[F(x ; \xi)]^{k+v+i}\right] d x, \\
E\left(X_{r: k}^{n}\right)= & \frac{\sum_{i, v=0}^{n-r}\left(\begin{array}{c}
k-r \\
v
\end{array}\right)(-1)^{v}}{B(r, k-r+1)}\left[\psi_{i, v} \varphi_{n, k+v+i-1}+\psi_{i, v}^{\prime} \varphi_{n, k+v+i}^{\prime}\right],
\end{aligned}
$$

where,

$$
\begin{aligned}
\varphi_{n, k+v+i-1} & =\int_{-\infty}^{\infty} x_{r: k}^{n} f(x ; \xi)[F(x ; \xi)]^{k+v+i-1} d x, \\
\varphi_{n, k+v+i}^{\prime} & =\int_{-\infty}^{\infty} x_{r: k}^{n} f(x ; \xi)[F(x ; \xi)]^{k+v+i} d x .
\end{aligned}
$$




\section{Special models}

Most of the extended forms of the statistical distributions are proposed for one of the following reasons: a generalization of an appropriate model that has formerly been used effectively, a model whose analytical fit is better to data, and to improve the characteristics of the existing model. Here, we provide some special distributions of the proposed family that can have at least one of these reasons.

\subsection{A new alpha power transformed Frechet distribution}

The cdf of the Frechet random variable is given by $F(x ; \xi)=e^{-(\gamma / x)^{\theta}}, x>0$, where $\xi=(\gamma, \theta)^{\top}$. The corresponding pdf is $f(x ; \xi)=\theta \gamma^{\theta} x^{-(\theta+1)} e^{-(\gamma / x)^{\theta}}$. The cdf and pdf of the new alpha power transformed Frechet (NAPTFr) distribution are given by

$$
G(x ; \Theta)=\frac{e^{-(\gamma / x)^{\Theta}} \alpha^{e^{-(\gamma / x)^{\Theta}}}}{\alpha}
$$

and

$$
g(x ; \Theta)=\frac{\theta \gamma^{\theta} x^{-(\theta+1)} e^{-(\gamma / x)^{\theta}} \alpha^{e^{-(\gamma / x)^{\theta}}}}{\alpha}\left[1+(\log \alpha) e^{-(\gamma / x)^{\theta}}\right],
$$

respectively. The plots for the pdf and hrf of the NAPTFr are sketched in Figure 1.

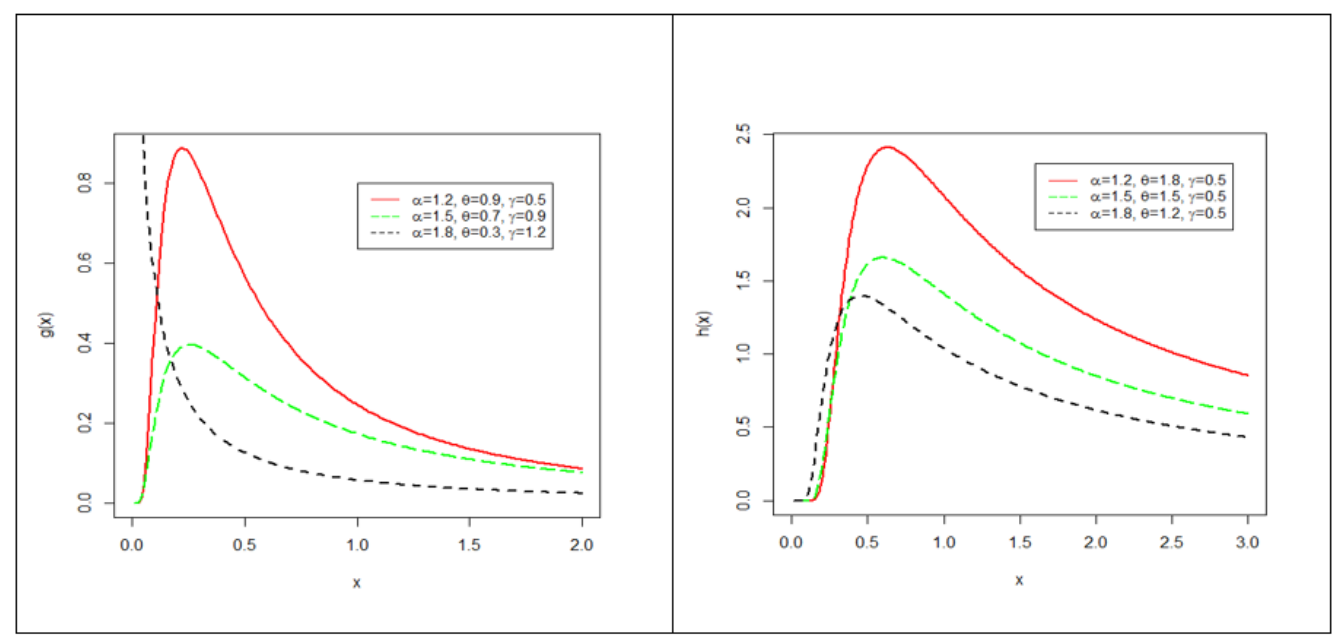

Figure 1: Different plots of pdf and hrf of NAPTFr distribution.

\subsection{A new alpha power transformed Pareto distribution}

Let $X$ follow the Pareto distribution with $\operatorname{cdf} F(x ; \xi)=1-(x / \gamma)^{-\theta}, x>\gamma$, and pdf $f(x ; \xi)=$ $(\theta / \gamma)(x / \gamma)^{-\theta-1}$, where $\xi=(\gamma, \theta)^{\top}$. The cdf of the new alpha power transformed Pareto (NAPTPa) distribution becomes

$$
G(x ; \Theta)=\frac{\left(1-(x / \gamma)^{-\theta}\right) \alpha^{(x / \gamma)^{-\theta}}}{\alpha} .
$$

The corresponding density function is

$$
g(x ; \Theta)=\frac{(\theta / \gamma)(x / \gamma)^{-\theta-1} \alpha^{(x / \gamma)^{-\theta}}}{\alpha}\left[1+(\log \alpha)\left(1-(x / \gamma)^{-\theta}\right)\right] .
$$

The visual representations of the pdf and hrf of the NAPTPa distribution are sketched in Figure 2. 


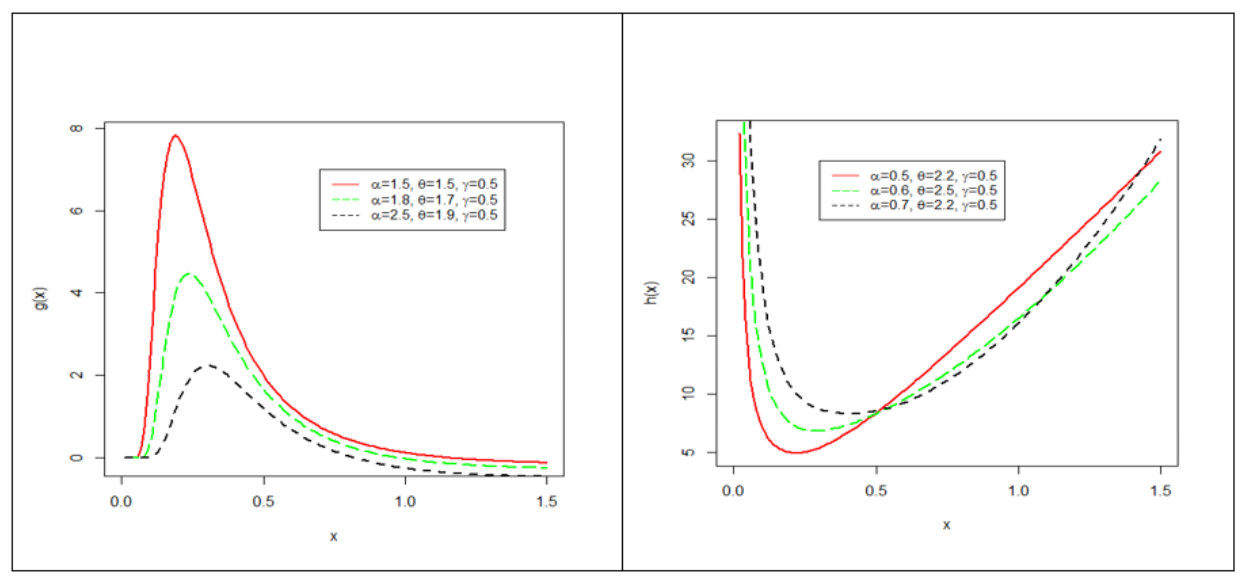

Figure 2: Different plots for the pdf and hrf of the NAPTPa distribution.

\subsection{A new alpha power transformed Lomax distribution}

Consider the case of Lomax random variable with cdf and pdf are given by $\mathrm{G}(x ; \xi)=1-(1+\gamma x)^{-\theta}$, and $g(x ; \xi)=\theta \gamma(1+\gamma x)^{-(\theta+1)}$, where $\xi=(\gamma, \theta)^{\top}$. Then, the cdf of the new alpha power transformed Lomax (NAPTL) distribution is given by

$$
G(x ; \Theta)=\frac{1-(1+\gamma x)^{-\theta}}{\alpha^{(1+\gamma x)^{-\theta}}} .
$$

The pdf NAPTL distribution is expressed by

$$
g(x ; \Theta)=\frac{\theta \gamma(1+\gamma x)^{-(\theta+1)}}{\alpha^{(1+\gamma x)^{-\theta}}}\left[1+(\log \alpha)\left(1-(1+\gamma x)^{-\theta}\right)\right] .
$$

Visual representations of the pdf and hrf of NAPTL distribution are presented in Figure 3.

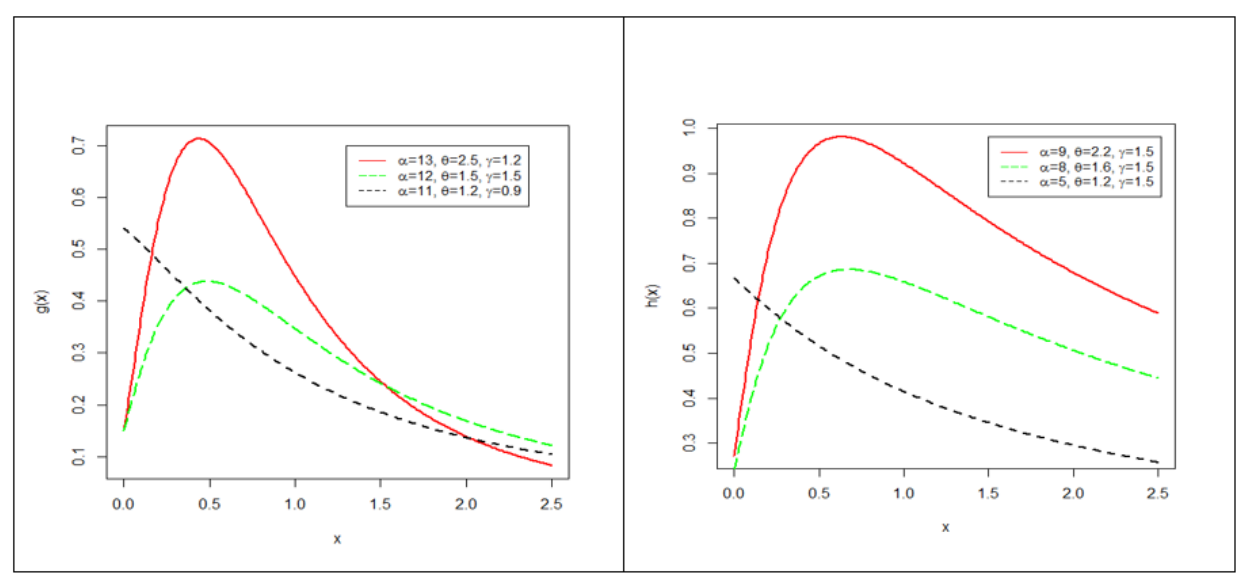

Figure 3: Different plots of the pdf and hrf of the NAPTL distribution.

\subsection{A new power transformed Weibull distribution}

The cdf and pdf of the Weibull distributed random variable are given as $F(x ; \zeta)=1-e^{-\gamma x^{\theta}}, x, \theta, \gamma>0$, and $f(x ; \zeta)=\gamma \theta x^{\theta-1} e^{-\gamma x^{\theta}}$, respectively, where $\xi=(\theta, \gamma)$. Then, cdf of the NAPTW model is given by

$$
G(x ; \Theta)=\frac{\left(1-e^{-\gamma x^{\Theta}}\right) \alpha^{\left(1-e^{-\gamma x^{\Theta}}\right)}}{\alpha}, \quad x, \alpha, \theta, \gamma>0, \alpha \neq 1 .
$$


The pdf, sf, hrf, rhrf, and chrf of NAPTW distribution are given in (5.1)-(5.5), respectively.

$$
\begin{aligned}
& g(x ; \Theta)=\frac{\gamma \theta x^{\theta-1} e^{-\gamma x^{\Theta}} \alpha^{\left(1-e^{-\gamma x^{\Theta}}\right)}}{\alpha}\left[1+\log (\alpha)\left(1-e^{-\gamma x^{\theta}}\right)\right], \\
& S(x ; \Theta)=\frac{\alpha-\left(1-e^{-\gamma x^{\theta}}\right) \alpha^{\left(1-e^{-\gamma x^{\theta}}\right)}}{\alpha}, \\
& h(x ; \Theta)=\frac{\gamma \theta x^{\theta-1} e^{-\gamma x^{\theta}}}{\alpha-\left(1-e^{-\gamma x^{\theta}}\right)}\left[1+\log (\alpha)\left(1-e^{-\gamma x^{\theta}}\right)\right], \\
& r(x ; \Theta)=\frac{\gamma \theta x^{\theta-1} e^{-\gamma x^{\theta}} \alpha^{\left(1-e^{-\gamma x^{\theta}}\right)}\left[1+\log (\alpha)\left(1-e^{-\gamma x^{\theta}}\right)\right]}{1-e^{-\gamma x^{\theta}}}, \\
& H(x ; \Theta)=-\log \left(\frac{\alpha-\left(1-e^{-\gamma x^{\Theta}}\right) \alpha^{\left(1-e^{-\gamma x^{\Theta}}\right)}}{\alpha}\right) .
\end{aligned}
$$

Figures 4 and 5 show the graphical sketching of the density and hrf's of the NAPTW distribution, for different parametric values.

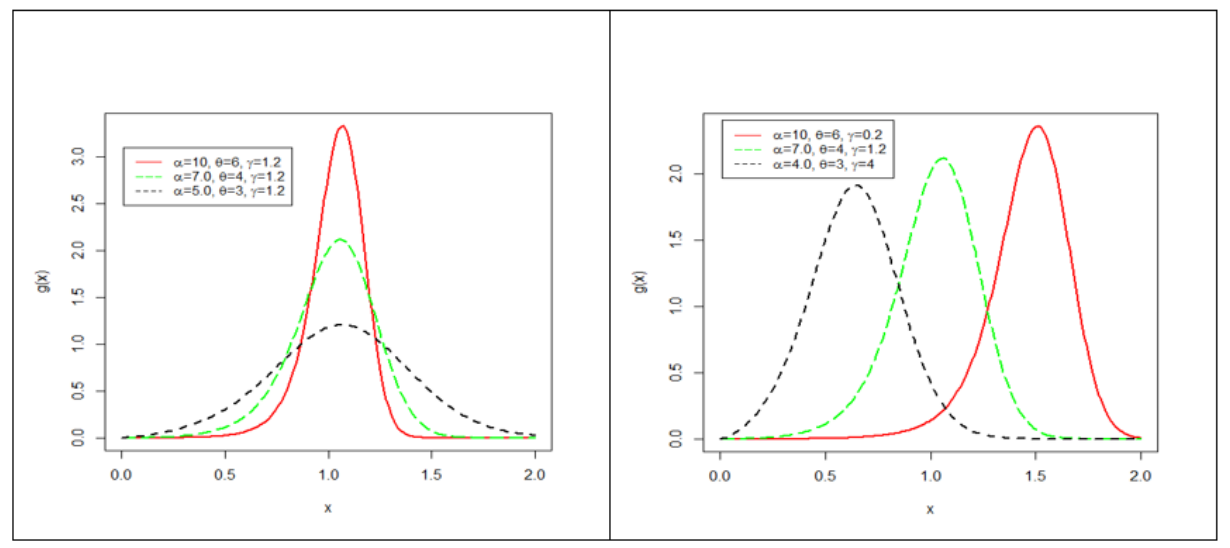

Figure 4: Density functions of NAPTW distribution, for different values of parameters.

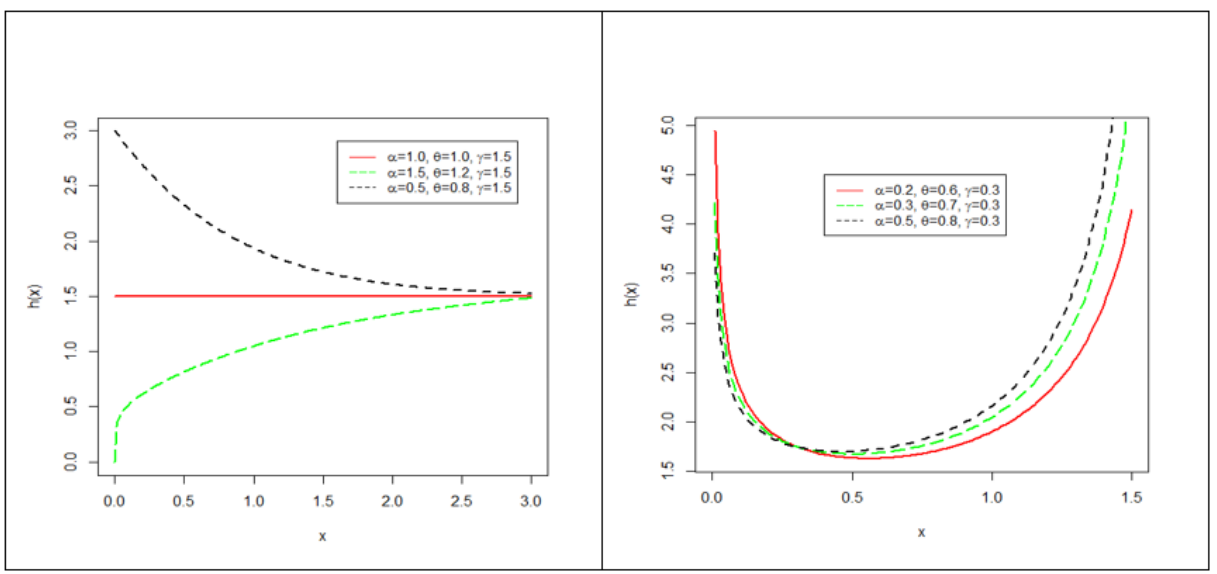

Figure 5: Hazard rate functions of NAPTW distribution, for selected parametric values. 
For the practical illustration of the proposed family we consider NAPTW distribution to analyze real life data. Let $X$ follows the NAPTW distribution, then $X$ reduces to

1. Weibull distribution, if $\alpha \rightarrow 1$;

2. one parameter Weibull distribution, if $\alpha \rightarrow 1$ and $\gamma=1$;

3. exponential distribution, if $\alpha \rightarrow 1$ and $\theta=1$;

4. Rayleigh distribution, if $\alpha \rightarrow 1$ and $\theta=2$;

5. one parameter NAPTW, if $\gamma=1$; (New)

6. NAPT exponential distribution, if $\theta=1$; (New)

7. NAPT Rayleigh distribution, if $\theta=2$. (New)

\section{Maximum likelihood estimation and simulation}

In this section, we provide maximum likelihood estimation of parameters and simulation of NAPT family.

\subsection{Maximum likelihood estimation}

This section concerns with the maximum likelihood estimation of parameters of the NAPT family based on complete set of samples. Let $X_{1}, X_{2}, \ldots, X_{k}$ be a random sample from NAPT family with parameters vector $\Theta$. The log-likelihood function of this sample is

$$
\log L=l=-k \log \alpha+\sum_{i=1}^{k} \log [f(x ; \xi)]+\sum_{i=1}^{k} \log \alpha[F(x ; \xi)]+\sum_{i=1}^{k} \log [1+\log (\alpha) F(x ; \xi)] .
$$

Obtaining the partial derivatives of (6.1), may get

$$
\begin{aligned}
\frac{\partial l}{\partial \alpha} & =-\frac{k}{\alpha}+\frac{1}{\alpha} \sum_{i=1}^{k} F(x ; \xi)+\frac{1}{\alpha} \sum_{i=1}^{k} \frac{F(x ; \xi)}{[1+\log \alpha F(x ; \xi)]}, \\
\frac{\partial l}{\partial \xi} & =\sum_{i=1}^{k} \frac{[\partial(f(x ; \xi)) / \partial \xi]}{f(x ; \xi)}+\sum_{i=1}^{k} \log \alpha[\partial(F(x ; \xi)) / \partial \xi]+\sum_{i=1}^{k} \frac{\log (\alpha)[\partial(F(x ; \xi)) / \partial \xi]}{[1+\log (\alpha) F(x ; \xi)]} .
\end{aligned}
$$

Setting (6.2) and (6.3) equal to zero and solving these equations simultaneously yield the maximum likelihood estimate (mle) $\hat{\Theta}=(\hat{\alpha}, \hat{\xi})$ of $\Theta=(\alpha, \xi)^{\top}$.

\subsection{Simulation study}

In this section, we provide simulation study to evaluate the performance of the maximum likelihood estimates (mle's) of the NAPT family. The algorithm used in this credit is as follow:

1. a random sample $X_{1}, X_{2}, \ldots, X_{k}$ of sizes $n=(50,100,150,200)$ are generated from the NAPT family;

2. select initial value for parameters;

3. for each sample sizes the estimates are obtained;

4. for each sample, steps (i)-(iii) are executed 1000 times, and mle's of the parameters, their biases and mean square errors are recorded.

The simulation results based on complete sample are provided in Table 1.

From the Table 1, it can easily be detected that

1. as the sample size increases, bias tend to decreases, which shows the accuracy of the maximum likelihood estimates;

2. also, as the sample size increases, MSE tend to decreases. That shows the consistency of these estimators.

The results obtained in Table 1, are graphically supported by Figures 6-9. 
Table 1: The parameter estimation from NAPTW distribution using maximum likelihood method.

\begin{tabular}{|c|c|c|c|c|c|c|c|c|c|}
\hline $\mathrm{n}$ & Par & Init & MLE & Bais & MSE & Init & MLE & Bais & MSE \\
\hline \multirow{3}{*}{30} & $\alpha$ & 0.5 & 0.5151 & 0.0151 & 0.0086 & 0.5 & 0.5156 & 0.0156 & 0.0087 \\
\cline { 2 - 10 } & $\theta$ & 0.5 & 0.5261 & 0.0261 & 0.0187 & 0.5 & 0.5233 & 0.0233 & 0.0165 \\
\cline { 2 - 10 } & $\gamma$ & 0.5 & 0.5134 & 0.0134 & 0.0072 & 0.75 & 0.7710 & 0.0210 & 0.0163 \\
\hline \multirow{3}{*}{50} & $\alpha$ & 0.5 & 0.5079 & 0.0079 & 0.0054 & 0.5 & 0.5081 & 0.0088 & 0.0046 \\
\cline { 2 - 10 } & $\theta$ & 0.5 & 0.5221 & 0.0221 & 0.0108 & 0.5 & 0.5113 & 0.0113 & 0.0091 \\
\cline { 2 - 10 } & $\gamma$ & 0.5 & 0.5075 & 0.0075 & 0.0046 & 0.75 & 0.7601 & 0.0101 & 0.0088 \\
\hline \multirow{4}{*}{100} & $\alpha$ & 0.5 & 0.5030 & 0.0030 & 0.0022 & 0.5 & 0.5077 & 0.0071 & 0.0018 \\
\cline { 2 - 9 } & $\theta$ & 0.5 & 0.5131 & 0.0131 & 0.0053 & 0.5 & 0.5051 & 0.0051 & 0.0044 \\
\cline { 2 - 9 } & $\gamma$ & 0.5 & 0.5022 & 0.0022 & 0.0019 & 0.75 & 0.7599 & 0.0099 & 0.0042 \\
\hline \multirow{3}{*}{30} & $\alpha$ & 0.5 & 0.5187 & 0.0187 & 0.0087 & 1.5 & 1.5087 & 0.0087 & 0.0201 \\
\cline { 2 - 9 } & $\theta$ & 0.5 & 0.5772 & 0.0772 & 0.0418 & 0.5 & 0.5130 & 0.0130 & 0.0151 \\
\cline { 2 - 9 } & $\gamma$ & 1.5 & 1.5493 & 0.0493 & 0.0670 & 1.5 & 1.5588 & 0.0588 & 0.1206 \\
\hline \multirow{3}{*}{50} & $\alpha$ & 0.5 & 0.5106 & 0.0106 & 0.0058 & 1.5 & 1.4997 & -0.0003 & 0.0107 \\
\cline { 2 - 9 } & $\theta$ & 0.5 & 0.5692 & 0.0692 & 0.0251 & 0.5 & 0.5043 & 0.0043 & 0.0087 \\
\cline { 2 - 9 } & $\gamma$ & 1.5 & 1.5274 & 0.0274 & 0.0440 & 1.5 & 1.5223 & 0.0223 & 0.0618 \\
\hline \multirow{3}{*}{100} & $\alpha$ & 0.5 & 0.5062 & 0.0062 & 0.0025 & 1.5 & 1.5021 & 0.0021 & 0.0052 \\
\cline { 2 - 9 } & $\theta$ & 0.5 & 0.5535 & 0.0535 & 0.0116 & 0.5 & 0.4939 & -0.0061 & 0.0037 \\
\cline { 2 - 9 } & $\gamma$ & 1.5 & 1.5176 & 0.0176 & 0.0190 & 1.5 & 1.5169 & 0.0169 & 0.0290 \\
\hline
\end{tabular}

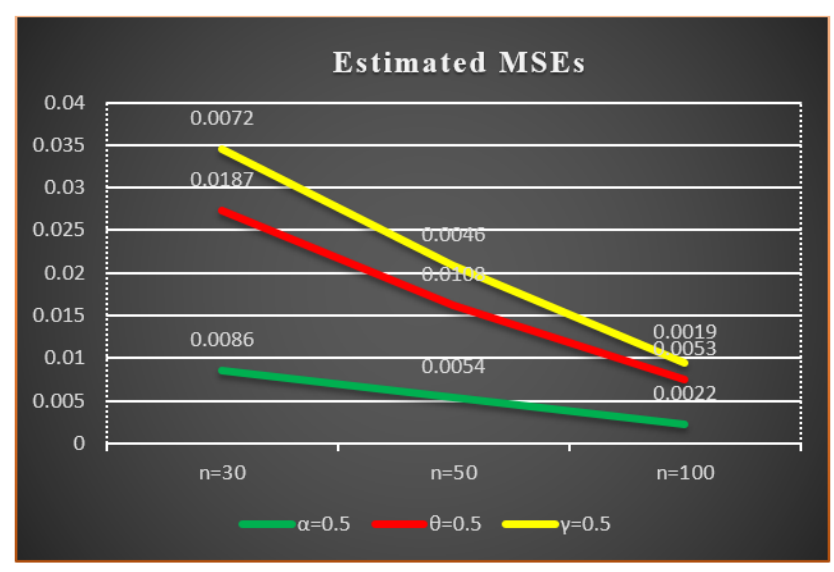

Figure 6: Estimated MSEs of the maximum likelihood estimators of NAPTW distribution.

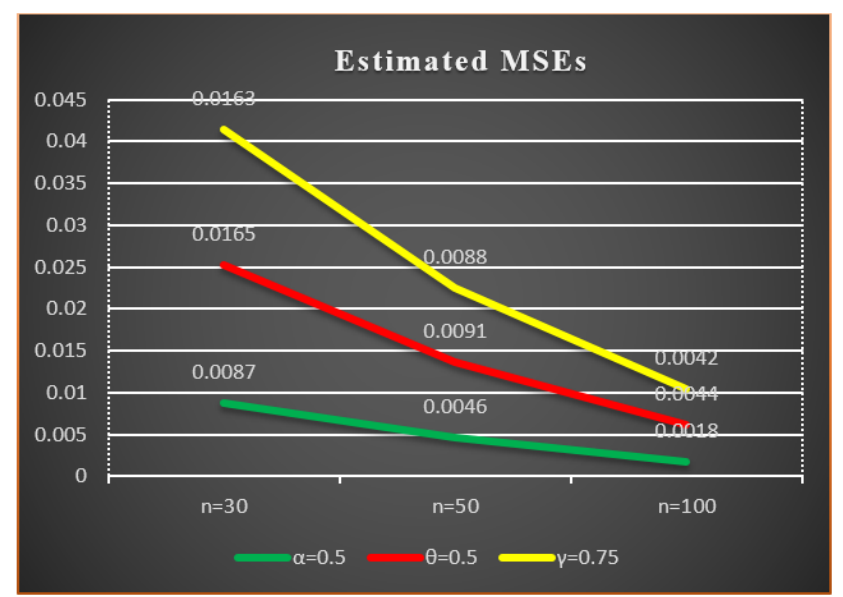

Figure 7: Estimated MSEs of the maximum likelihood estimators of NAPTW distribution. 


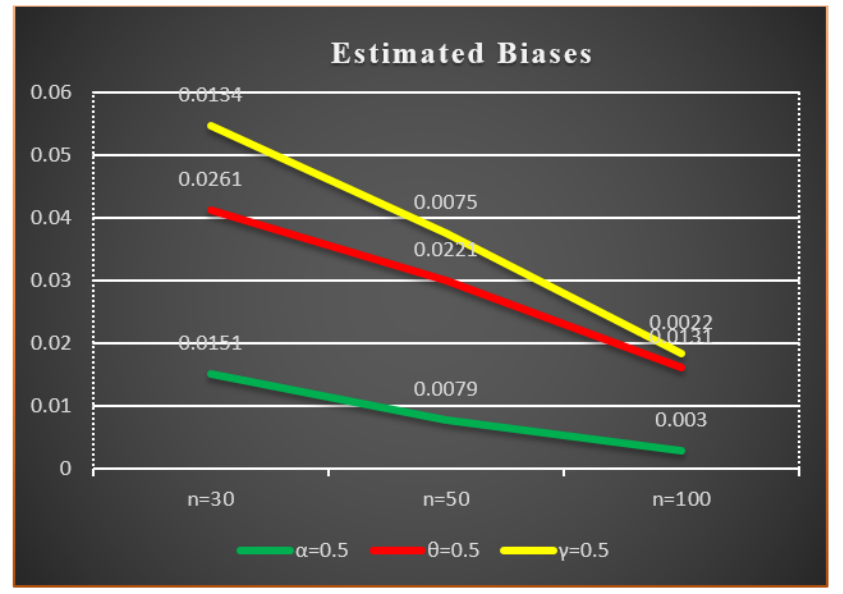

Figure 8: Estimated Biases of the maximum likelihood estimators of NAPTW distribution.

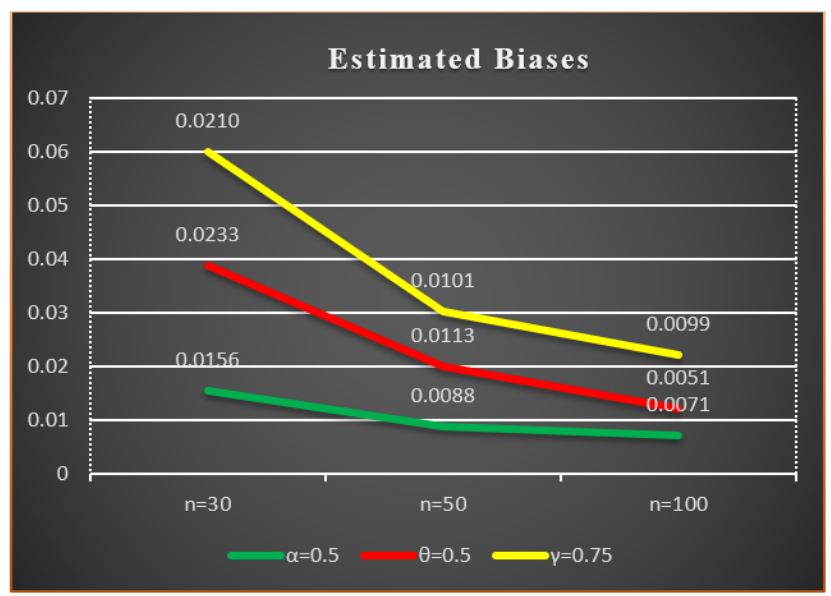

Figure 9: Estimated Biases of the maximum likelihood estimators of NAPTW distribution.

\section{Estimation under censoring samples}

In reliability or lifetime testing experiments, the mostly data are censored due to various reasons such as time limitation, cost or other resources. There are two common censoring schemes known as Type-I and Type-II censoring schemes. Here we discuss estimation based on these two censoring schemes. In Type-I censoring, we have a fixed time say $X$ but the number of items fail during the experiment is random. Whereas, in Type-II censoring scheme, the experiment is continued (i.e., time varies) until the specified number of failures occur.

\subsection{Type-I censoring estimation}

Suppose that $X_{1}, X_{2}, \ldots, X_{r}$ be a type-I censoring sample of size $r$ obtained from lifetime testing experiment on $k$ items whose lifetime following the density given in (1.3). The likelihood function of Type-I censoring sampling is given

$$
L(\alpha, \xi)=\left(\begin{array}{l}
k \\
r
\end{array}\right) \frac{1}{\alpha^{r}} \prod_{i=1}^{r} f\left(x_{i} ; \xi\right) \prod_{i=1}^{r} \alpha^{F\left(x_{i} ; \xi\right)} \prod_{i=1}^{r}\left[1+(\log \alpha) F\left(x_{i} ; \xi\right)\right]\left(\frac{\alpha-F(X ; \xi) \alpha^{F(X ; \xi)}}{\alpha}\right)^{k-r},
$$


the log-likelihood function (LL) corresponding to (7.1) is given by

$$
\begin{aligned}
L(\alpha, \xi)=l= & -r \log \alpha+\log \left(\begin{array}{c}
k \\
r
\end{array}\right)+\sum_{i=1}^{r} \log \left[f\left(x_{i} ; \xi\right)\right]+\sum_{i=1}^{r}(\log \alpha)\left[F\left(x_{i} ; \xi\right)\right] \\
& +\sum_{i=1}^{r} \log \left[1+(\log \alpha) F\left(x_{i} ; \xi\right)\right]+(k-r) \log \left(\frac{\alpha-F(X ; \xi) \alpha^{F(X ; \xi)}}{\alpha}\right) .
\end{aligned}
$$

Obtaining the partial derivatives of (7.2), we get

$$
\begin{aligned}
\frac{\partial l}{\partial \alpha}= & -\frac{r}{\alpha}+\frac{1}{\alpha} \sum_{i=1}^{r} F\left(x_{i} ; \xi\right)+\frac{1}{\alpha} \sum_{i=1}^{r} \frac{F\left(x_{i} ; \xi\right)}{\left[1+(\log \alpha) F\left(x_{i} ; \xi\right)\right]} \\
& +(k-r) \frac{F(X ; \xi) \alpha^{F(X ; \xi)}}{\alpha}\left\{\frac{[1-F(X ; \xi)]}{\left(\alpha-F(X ; \xi) \alpha^{F(X ; \xi)}\right)}\right\}, \\
\frac{\partial l}{\partial \xi}= & \sum_{i=1}^{r} \frac{\left[\partial\left(f\left(x_{i} ; \xi\right)\right) / \partial \xi\right]}{f\left(x_{i} ; \xi\right)}+\sum_{i=1}^{r}(\log \alpha)\left[\partial\left(F\left(x_{i} ; \xi\right)\right) / \partial \xi\right]+\sum_{i=1}^{r} \frac{(\log \alpha)\left[\partial\left(F\left(x_{i} ; \xi\right)\right) / \partial \xi\right]}{\left[1+(\log \alpha) F\left(x_{i} ; \xi\right)\right]} \\
& -(k-r) \frac{\{\partial(F(X ; \xi)) / \partial \xi\} \alpha^{F(X ; \xi)}[1+(\log \alpha) F(X ; \xi)]}{\left[\alpha-F(X ; \xi) \alpha^{F(X ; \xi)}\right]}
\end{aligned}
$$

\begin{tabular}{|c|c|c|c|c|c|c|c|c|c|c|}
\hline k & Par & $\mathrm{t}_{0}$ & Init & MLE & Bais & MSE & Init & MLE & Bais & MSE \\
\hline \multirow{6}{*}{30} & \multirow[t]{2}{*}{$\gamma$} & 10 & \multirow[t]{2}{*}{0.5} & 0.5093 & 0.0093 & 0.0075 & \multirow[t]{2}{*}{0.75} & 0.7693 & 0.0193 & 0.0159 \\
\hline & & 20 & & 0.5103 & 0.0103 & 0.0078 & & 0.7672 & 0.0172 & .0160 \\
\hline & \multirow[t]{2}{*}{$\theta$} & 10 & \multirow[t]{2}{*}{0.5} & 0.5301 & 0.0301 & 0.0197 & \multirow[t]{2}{*}{0.5} & 0.5210 & 0.0210 & 0.0202 \\
\hline & & 20 & & 0.5262 & 0.0262 & 0.0185 & & 0.5194 & 0.0194 & 0.0169 \\
\hline & \multirow[t]{2}{*}{$\alpha$} & 10 & \multirow[t]{2}{*}{0.5} & 0.5118 & 0.0118 & 0.0093 & \multirow[t]{2}{*}{0.5} & 0.5150 & 0.0150 & 0.0085 \\
\hline & & 20 & & 0.5123 & 0.0123 & 0.0093 & & 0.5136 & 0.0136 & 0.0085 \\
\hline \multirow{6}{*}{50} & & 10 & \multirow[t]{2}{*}{0.5} & 0.5056 & 0.0056 & 0.0045 & \multirow[t]{2}{*}{0.75} & 0.7541 & 0.0041 & 0.0087 \\
\hline & & 20 & & 0.5077 & 0.0077 & 0.0044 & & 0.7651 & 0.0151 & 0.0090 \\
\hline & \multirow[t]{2}{*}{$\theta$} & 10 & \multirow[t]{2}{*}{0.5} & 0.5085 & 0.0085 & 0.0090 & \multirow[t]{2}{*}{0.5} & 0.5046 & 0.0046 & 0.0085 \\
\hline & & 20 & & 0.5157 & 0.0157 & 0.0105 & & 0.5094 & 0.0094 & 0.0095 \\
\hline & & 10 & \multirow[t]{2}{*}{0.5} & 0.5068 & 0.0068 & 0.0055 & \multirow[t]{2}{*}{0.5} & 0.5035 & 0.0035 & 0.0046 \\
\hline & & 20 & & 0.5094 & 0.0094 & 0.0054 & & 0.5116 & 0.0116 & 0.0048 \\
\hline \multirow{6}{*}{100} & \multirow[t]{2}{*}{$\gamma$} & 10 & \multirow[t]{2}{*}{0.5} & 0.5038 & 0.0038 & 0.0023 & \multirow[t]{2}{*}{0.75} & 0.7531 & 0.0031 & 0.0046 \\
\hline & & 20 & & 0.5013 & 0.0013 & 0.0022 & & 0.7555 & 0.0055 & 0.0048 \\
\hline & \multirow[t]{2}{*}{$\theta$} & 10 & \multirow[t]{2}{*}{0.5} & 0.5056 & 0.0056 & 0.0045 & \multirow[t]{2}{*}{0.5} & 0.5023 & 0.0023 & 0.0045 \\
\hline & & 20 & & 0.5086 & 0.0086 & 0.0045 & & 0.5022 & 0.0022 & 0.0044 \\
\hline & & 10 & \multirow[t]{2}{*}{0.5} & 0.5046 & 0.0046 & 0.0027 & \multirow[t]{2}{*}{0.5} & 0.5027 & 0.0027 & 0.0024 \\
\hline & & 20 & & 0.5019 & 0.0019 & 0.0026 & & 0.5044 & 0.0044 & 0.0025 \\
\hline
\end{tabular}

By equating (7.3) and (7.4) to zero and solving simultaneously we have the mle's $\hat{\Theta}=(\hat{\alpha}, \hat{\xi})$ of $\Theta=(\alpha, \xi)^{\top}$ based on Type-I censored sample. Simulation results based on Type-I censoring samples for the NAPTW distribution are provided in Table 2.

Table 2: The parameter estimation from NAPTW distribution using maximum likelihood method under Type-I censored samples.

From Table 2, it is quite clear that as $k$ increases then Bais and MSE of the estimator's decrease.

\subsection{Type-II censoring estimation}

Consider $X_{1}, X_{2}, \ldots, X_{r}$ be a type-II censoring sample of size $r$ observed from lifetime testing experiment on $k$ items whose lifetime have the density expressed in (1.3). The likelihood function of type-II 
censoring sampling is

$$
L(\alpha, \xi)=\frac{1}{\alpha^{r}}\left(\begin{array}{l}
k \\
r
\end{array}\right) \prod_{i=1}^{r} f\left(x_{i} ; \xi\right) \prod_{i=1}^{r} \alpha^{F\left(x_{i} ; \xi\right)} \prod_{i=1}^{r}\left[1+(\log \alpha) F\left(x_{i} ; \xi\right)\right]\left(\frac{\alpha-F\left(x_{r} ; \xi\right) \alpha^{F\left(x_{r} ; \xi\right)}}{\alpha}\right)^{k-r} .
$$

The LL estimates of the mle's $\hat{\Theta}=(\hat{\alpha}, \hat{\xi})$ of $\Theta=(\alpha, \xi)^{\top}$ based on Type-II censored sample can be obtained by solving the following non-linear equations:

$$
\begin{aligned}
\frac{\partial l}{\partial \alpha}= & -\frac{r}{\alpha}+\frac{1}{\alpha} \sum_{i=1}^{r} F\left(x_{i} ; \xi\right)+\frac{1}{\alpha} \sum_{i=1}^{r} \frac{F\left(x_{i} ; \xi\right)}{\left[1+(\log \alpha) F\left(x_{i} ; \xi\right)\right]} \\
& +(k-r) \frac{F\left(x_{r} ; \xi\right) \alpha^{F\left(x_{r} ; \xi\right)}}{\alpha}\left\{\frac{\left[1-F\left(x_{r} ; \xi\right)\right]}{\left(\alpha-F\left(x_{r} ; \xi\right) \alpha^{F\left(x_{r} ; \xi\right)}\right)}\right\}, \\
\frac{\partial l}{\partial \xi}= & \sum_{i=1}^{r} \frac{\left[\partial\left(f\left(x_{i} ; \xi\right)\right) / \partial \xi\right]}{f\left(x_{i} ; \xi\right)}+\sum_{i=1}^{r}(\log \alpha)\left[\partial\left(F\left(x_{i} ; \xi\right)\right) / \partial \xi\right]+\sum_{i=1}^{r} \frac{(\log \alpha)\left[\partial\left(F\left(x_{i} ; \xi\right)\right) / \partial \xi\right]}{\left[1+(\log \alpha) F\left(x_{i} ; \xi\right)\right]} \\
& -(k-r) \frac{\left\{\partial\left(F\left(x_{i} ; \xi\right)\right) / \partial \xi\right\} \alpha^{F\left(x_{r} ; \xi\right)}\left[1+(\log \alpha) F\left(x_{r} ; \xi\right)\right]}{\left[\alpha-f\left(x_{r} ; \xi\right) \alpha^{F\left(x_{r} ; \xi\right)}\right]}
\end{aligned}
$$

\begin{tabular}{|c|c|c|c|c|c|c|c|c|c|c|}
\hline k & Par & $\mathrm{X}_{\mathrm{r}}$ & Init & MLE & Bais & MSE & Init & MLE & Bais & MSE \\
\hline \multirow{6}{*}{100} & \multirow[t]{2}{*}{$\gamma$} & $50 \%$ & \multirow[t]{2}{*}{0.5} & 0.5248 & 0.0248 & 0.0192 & \multirow[t]{2}{*}{0.75} & 0.7971 & 0.0471 & 0.0471 \\
\hline & & $80 \%$ & & 0.5192 & 0.0192 & 0.0096 & & 0.7748 & 0.0248 & 0.0220 \\
\hline & \multirow[t]{2}{*}{$\theta$} & $50 \%$ & \multirow[t]{2}{*}{0.5} & 0.5257 & 0.0257 & 0.0315 & \multirow[t]{2}{*}{0.5} & 0.5614 & 0.0614 & 0.0708 \\
\hline & & $80 \%$ & & 0.5273 & 0.0273 & 0.0199 & & 0.5226 & 0.0226 & 0.0204 \\
\hline & \multirow[t]{2}{*}{$\alpha$} & $50 \%$ & \multirow[t]{2}{*}{0.5} & 0.5278 & 0.0278 & 0.0215 & \multirow[t]{2}{*}{0.5} & 0.5351 & 0.0351 & 0.0237 \\
\hline & & $80 \%$ & & 0.5228 & 0.0228 & 0.0117 & & 0.5197 & 0.0197 & 0.0118 \\
\hline \multirow{6}{*}{300} & \multirow[t]{2}{*}{$\gamma$} & $50 \%$ & \multirow[t]{2}{*}{0.5} & 0.5182 & 0.0182 & 0.0102 & \multirow[t]{2}{*}{0.75} & 0.7773 & 0.0273 & 0.0233 \\
\hline & & $80 \%$ & & 0.5140 & 0.0140 & 0.0058 & & 0.7655 & 0.0155 & 0.0124 \\
\hline & \multirow[t]{2}{*}{$\theta$} & $50 \%$ & \multirow[t]{2}{*}{0.5} & 0.5034 & 0.0034 & 0.0159 & \multirow[t]{2}{*}{0.5} & 0.5305 & 0.0305 & 0.0305 \\
\hline & & $80 \%$ & & 0.5042 & 0.0042 & 0.0090 & & 0.5092 & 0.0092 & 0.0105 \\
\hline & \multirow[t]{2}{*}{$\alpha$} & $50 \%$ & 0.5 & 0.5201 & 0.0201 & 0.0115 & \multirow[t]{2}{*}{0.5} & 0.5204 & 0.0204 & 0.0116 \\
\hline & & $80 \%$ & & 0.5161 & 0.0161 & 0.0070 & & 0.5124 & 0.0124 & 0.0067 \\
\hline \multirow{6}{*}{100} & \multirow[t]{2}{*}{$\gamma$} & $50 \%$ & 0.5 & 0.5093 & 0.0093 & 0.0050 & \multirow[t]{2}{*}{0.75} & 0.7589 & 0.0089 & 0.0108 \\
\hline & & $80 \%$ & & 0.5058 & 0.0058 & 0.0027 & & 0.7614 & 0.0114 & 0.0062 \\
\hline & \multirow[t]{2}{*}{$\theta$} & $50 \%$ & 0.5 & 0.4925 & -0.0075 & 0.0068 & \multirow[t]{2}{*}{0.5} & 0.5093 & 0.0093 & 0.0123 \\
\hline & & $80 \%$ & & 0.4993 & -0.0007 & 0.0042 & & 0.5004 & 0.0004 & 0.0050 \\
\hline & \multirow[t]{2}{*}{0} & $50 \%$ & 0.5 & 0.5103 & 0.0103 & 0.0056 & \multirow[t]{2}{*}{0.5} & 0.5068 & 0.0068 & 0.0054 \\
\hline & & $80 \%$ & & 0.5069 & 0.0069 & 0.0032 & & 0.5089 & 0.0089 & 0.0033 \\
\hline
\end{tabular}

Simulation results based on Type-II censoring samples for the NAPTW distribution are provided in Table 3.

Table 3: The parameter estimation from NAPTW distribution using maximum likelihood method under Type-II censored samples.

From Table 3, it is quite clear that as k increases then Bais and MSE of the estimator's decrease.

\section{Applications}

In this section, we provide a comparison study of the proposed model and other existing distributions. We analyze fracture toughness data from the two different materials: the first material are Alumina 
(Al2O3) taken from the website: http://www.ceramics.nist.gov/srd/summary/ftmain.htm. This data set can also be found in Nadarajah and Kotz [20]. While, the second data set taken from Saboor and Pogány [24]. We fit a special model (called NAPTW distribution) of the proposed family in comparison with five other well-known competing distributions. The cumulative functions of the competing models are:

1. exponentiated Weibull (Ex-W) distribution introduced by Mudholkar and Srivastava [19]

$$
\mathrm{G}(x)=\left(1-e^{-\gamma x^{\theta}}\right)^{\alpha}, \quad x, \alpha, \theta, \gamma>0
$$

2. modified Weibull (MW) distribution of Lai et al. [16]

$$
G(x)=1-e^{-\gamma x^{\theta} e^{\alpha x}}, \quad x, \alpha, \theta, \gamma>0 ;
$$

3. Kumaraswamy Weibull $(\mathrm{Ku}-\mathrm{W})$ distribution by Cordeiro et al. [6]

$$
\mathrm{G}(x)=1-\left(1-\left(1-e^{-\gamma x^{\theta}}\right)^{a}\right)^{b}, \quad x, a, b, \theta, \gamma>0
$$

4. alpha power transformed Weibull (APTW) distribution of Dey et al. [7]

$$
G(x)=\frac{\alpha^{\left(1-e^{-\gamma x^{\theta}}\right)}-1}{\alpha-1}, \quad x, \alpha, \theta, \gamma>0, \quad \alpha \neq 1 ;
$$

5. flexible Weibull extended (FWE) distribution proposed by Ahmad and Hussain [1]

$$
G(x)=1-\exp \left(-e^{\alpha x^{2}-\frac{\gamma}{x^{\theta}}}\right), \quad x, \alpha, \theta, \gamma>0 .
$$

The analytical measure such as Kolmogorov-Smirnov (KS) test statistic, Cramer-von-Misses (CM) test statistic, Anderson-Darling (AD) test statistic, Akaike Information Criterion (AIC), Bayesian Information Criterion (BIC), corrected Akaike information criterion (CAIC), and Hannan-Quinn information criterion (HQIC) are considered for deciding the goodness of fit results of the proposed model and other competing models. On considering these measures, it is showed that the newly proposed model provides

\begin{tabular}{|c|c|}
\hline Data 1 Alumina (Al2O 3) & $\begin{array}{c}5.5,5,4.9,6.4,5.1,5.2,5.2,5,4.7,4,4.5,4.2,4.1,4.56,5.01,4.7,3.13,3.12,2.68, \\
2.77,2.7,2.36,4.38,5.73,4.35,6.81,1.91,2.66,2.61,1.68,2.04,2.08,2.13,3.8, \\
3.73,3.71,3.28,3.9,4,3.8,4.1,3.9,4.05,4,3.95,4,4.5,4.5,4.2,4.55,4.65,4.1, \\
4.25,4.3,4.5,4.7,5.15,4.3,4.5,4.9,5,5.35,5.15,5.25,5.8,5.85,5.9,5.75,6.25, \\
6.05,5.9,3.6,4.1,4.5,5.3,4.85,5.3,5.45,5.1,5.3,5.2,5.3,5.25,4.75,4.5,4.2,4, \\
4.15,4.25,4.3,3.75,3.95,3.51,4.13,5.4,5,2.1,4.6,3.2,2.5,4.1,3.5,3.2,3.3,4.6, \\
4.3,4.3,4.5,5.5,4.6,4.9,4.3,3,3.4,3.7,4.4,4.9,4.9,5\end{array}$ \\
\hline Data 2 Corban fibers data & $\begin{array}{c}3.70,2.74,2.73,2.50,3.60,3.11,3.27,2.87,1.47,3.11,3.56,4.42,2.41,3.19,3.22, \\
1.69,3.28,3.09,1.87,3.15,4.90,1.57,2.67,2.93,3.22,3.39,2.81,4.20,3.33,2.55, \\
3.31,3.31,2.85,1.25,4.38,1.84,0.39,3.68,2.48,0.85,1.61,2.79,4.70,2.03,1.89, \\
2.88,2.82,2.05,3.65,3.75,2.43,2.95,2.97,3.39,2.96,2.35,2.55,2.59,2.03,1.61, \\
2.12,3.15,1.08,2.56,1.80,2.53\end{array}$ \\
\hline
\end{tabular}
greater distributional flexibility than the other well-known lifetime distributions. For the interest of the researchers, we have provided the data in Table 4 . While, summary statistics of the data are mentioned in Table 5.

Table 4: Fracture toughness and Corban fibers data sets. 
Table 5: Summary of fracture toughness data.

\begin{tabular}{|c|c|c|c|c|c|c|}
\hline Summary Statistics & Min. & $1^{\text {st }}$ Quartile & Median & Mean & $3^{\text {rd }}$ Quartile & Max. \\
\hline Data 1 & 1.680 & 3.850 & 4.380 & 4.325 & 5.000 & 6.810 \\
\hline Data 2 & 0.390 & 2.178 & 2.835 & 2.760 & 3.278 & 4.900 \\
\hline
\end{tabular}

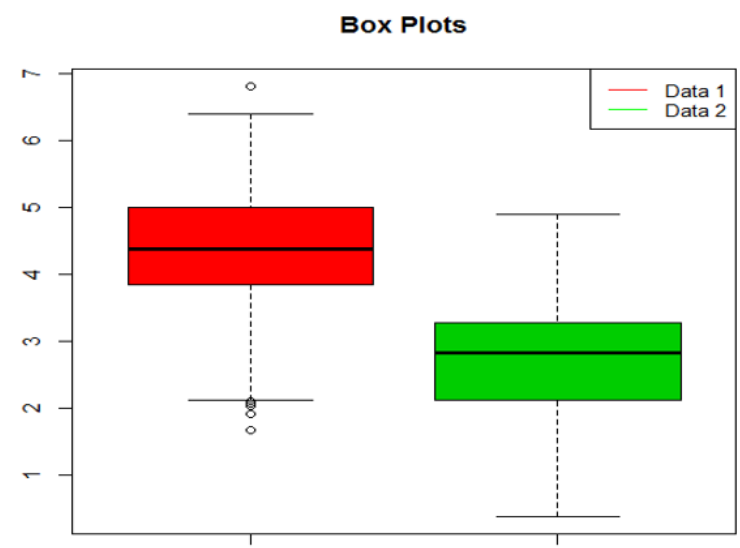

Figure 10: Box plots of the data sets provided in Table 1.

Table 6: Estimates of the parameters and standard errors in parentheses for the models fitted to material 1.

\begin{tabular}{|c|c|c|c|c|c|}
\hline Dist. & $\hat{\gamma}$ & $\hat{\theta}$ & $\hat{\alpha}$ & $\hat{\mathrm{a}}$ & $\hat{b}$ \\
\hline NAPTW & $0.006(0.0005)$ & $3.615(0.1298)$ & $4.932(0.6970)$ & & \\
\hline APTW & $0.003(0.0007)$ & $3.931(0.1318)$ & $4.958(0.7308)$ & & \\
\hline FWE & $5.961(1.0160)$ & $3.832(0.1719)$ & $4.073(0.7900)$ & & \\
\hline MW & $0.008(0.0006)$ & $3.873(0.7433)$ & $1.034(0.2206)$ & & \\
\hline Ex-W & $0.007(0.0022)$ & $3.620(0.1622)$ & $4.867(0.2739)$ & & \\
\hline Ku-W & $0.007(0.0070)$ & 3.657 (0.3609) & & $1.663(0.4281)$ & $2.301(2.6960)$ \\
\hline
\end{tabular}

Table 7: Analytical results of the proposed and other competing models for material 1.

\begin{tabular}{|c|c|c|c|c|c|c|c|c|}
\hline Dist. & KS & AD & CM & LL & AIC & BIC & CIAC & HQIC \\
\hline NAPTW & $\mathbf{0 . 0 4 8}$ & $\mathbf{0 . 3 8 9}$ & $\mathbf{0 . 0 6 0}$ & $\mathbf{8 5 . 3 9}$ & $\mathbf{3 4 2 . 5 1}$ & $\mathbf{3 5 0 . 8 5}$ & $\mathbf{3 4 2 . 7 2}$ & $\mathbf{3 4 5 . 9 0}$ \\
\hline APTW & 0.144 & 0.513 & 0.082 & 85.52 & 347.21 & 355.55 & 347.42 & 350.60 \\
\hline FWE & 0.109 & 0.731 & 0.126 & 171.97 & 349.95 & 358.29 & 350.16 & 353.34 \\
\hline MW & 0.079 & 0.675 & 0.111 & 171.71 & 349.43 & 357.77 & 349.64 & 352.82 \\
\hline Ex-W & 0.082 & 1.045 & 0.168 & 86.57 & 347.72 & 356.05 & 347.92 & 351.10 \\
\hline Ku-W & 0.059 & 0.757 & 0.121 & 86.24 & 348.10 & 359.21 & 348.45 & 352.61 \\
\hline
\end{tabular}

Table 8: Estimates of the parameters and standard errors in parentheses for the models fitted to material 2.

\begin{tabular}{|cccccc|}
\hline Dist. & $\hat{\gamma}$ & $\hat{\theta}$ & $\hat{\alpha}$ & $\hat{a}$ & $\hat{b}$ \\
\hline NAPTW & $0.065(0.0069)$ & $1.573(0.4498)$ & $4.159(0.4023)$ & & \\
\hline APTW & $0.059(0.0560)$ & $2.887(0.5718)$ & $5.091(7.6069)$ & & \\
\hline FWE & $3.063(0.4502)$ & $1.826(0.5753)$ & $0.115(0.0134)$ & & \\
\hline MW & $0.024(0.0093)$ & $2.468(1.9479)$ & $0.294(0.3676)$ & & \\
\hline Ex-W & $0.023(0.0249)$ & $3.362(0.6778)$ & $1.046(0.3743)$ & & \\
\hline Ku-W & $0.012(0.0073)$ & $2.917(0.4676)$ & & $1.235(0.2678)$ & $4.147(2.7288)$ \\
\hline
\end{tabular}




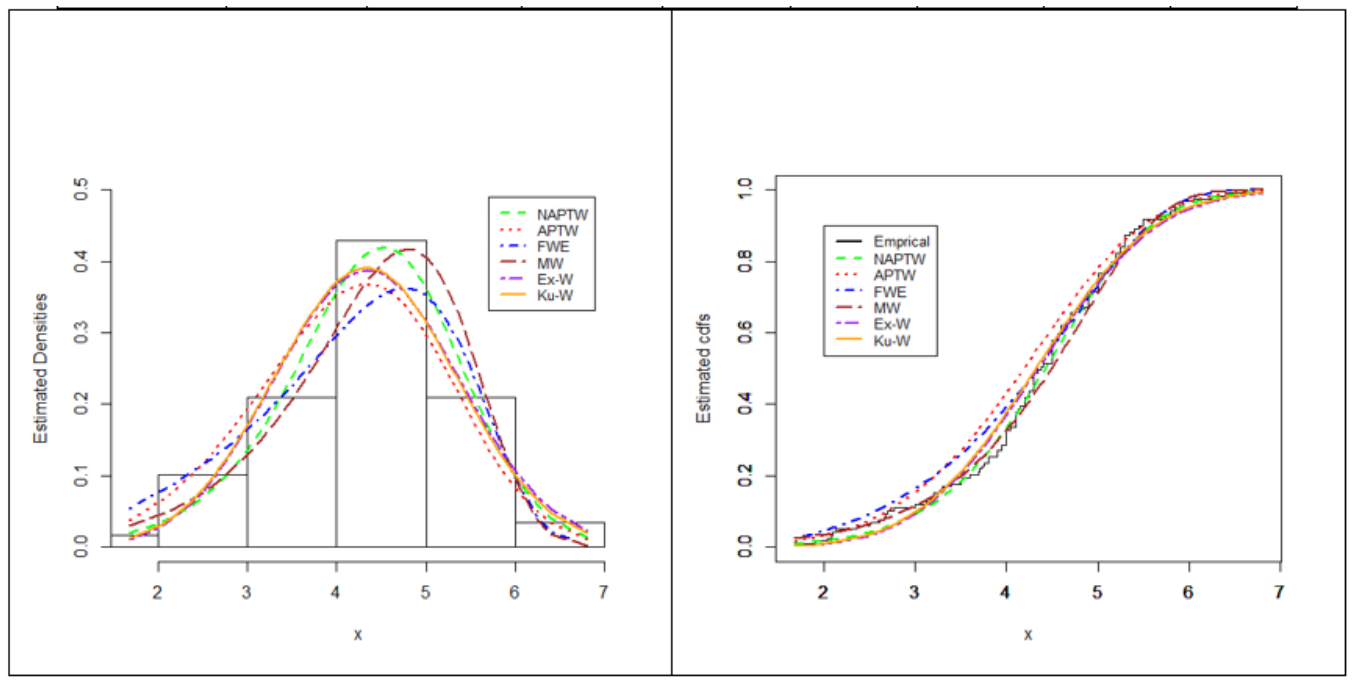

Figure 11: Estimated densities and cdfs of the fitted models for data 1.
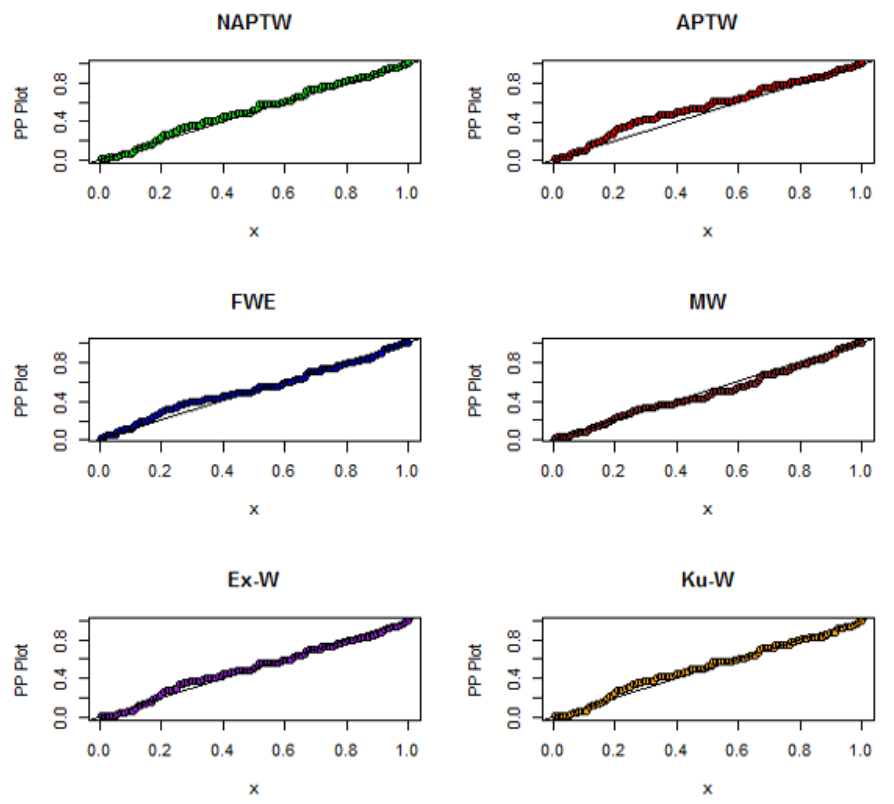

Figure 12: PP-plots of the fitted models for data 1.

Table 9: Analytical results of the proposed and other competing models for material 2.

\begin{tabular}{|c|c|c|c|c|c|c|c|c|}
\hline Dist. & KS & AD & CM & LL & AIC & BIC & CIAC & HQIC \\
\hline NAPTW & $\mathbf{0 . 0 7 0}$ & $\mathbf{0 . 3 8 7}$ & $\mathbf{0 . 0 6 7}$ & $\mathbf{8 5 . 2 4}$ & $\mathbf{1 7 6 . 4 9}$ & $\mathbf{1 8 3 . 0 6}$ & $\mathbf{1 7 6 . 8 8}$ & $\mathbf{1 7 9 . 0 9}$ \\
\hline APTW & 0.074 & 0.394 & 0.071 & 85.35 & 176.70 & 183.27 & 177.09 & 179.30 \\
\hline FWE & 0.124 & 1.135 & 0.175 & 89.60 & 185.21 & 191.78 & 185.60 & 187.80 \\
\hline MW & 0.089 & 0.496 & 0.081 & 85.93 & 177.86 & 184.43 & 178.25 & 180.46 \\
\hline Ex-W & 0.080 & 0.534 & 0.095 & 86.12 & 178.24 & 184.81 & 178.63 & 180.84 \\
\hline Ku-W & 0.082 & 0.551 & 0.099 & 86.24 & 180.48 & 189.24 & 181.13 & 183.94 \\
\hline
\end{tabular}




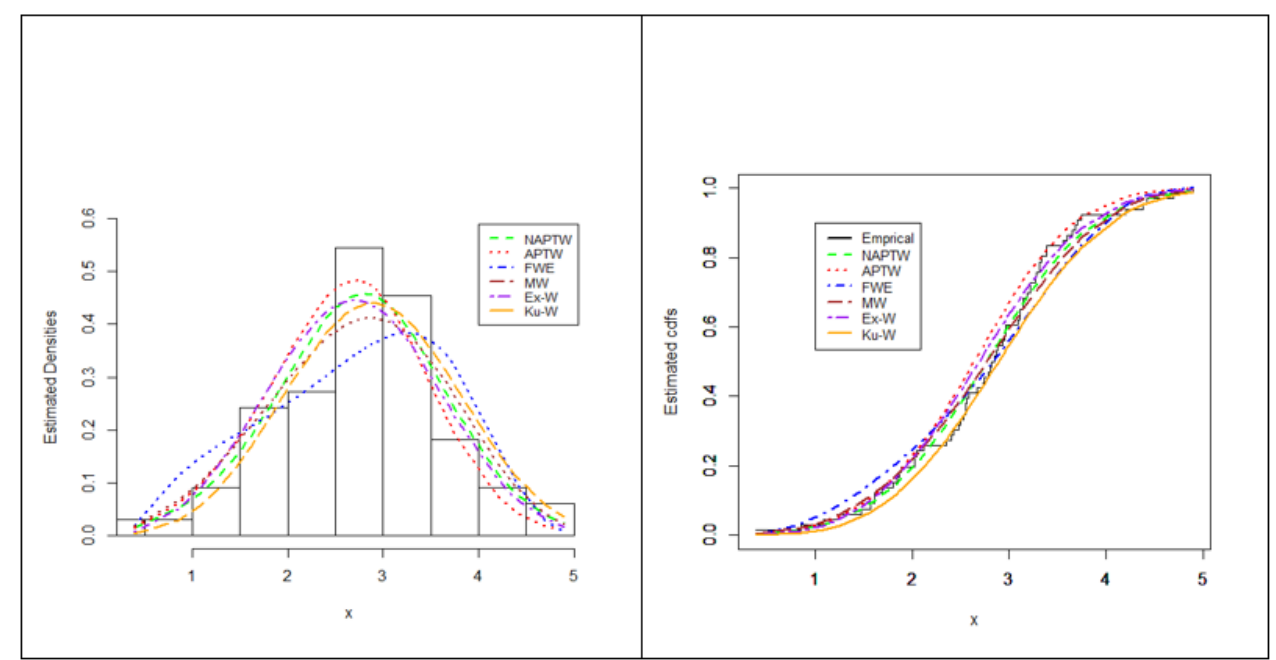

Figure 13: Estimated densities and cdfs of the fitted distributions corresponding to data 2.
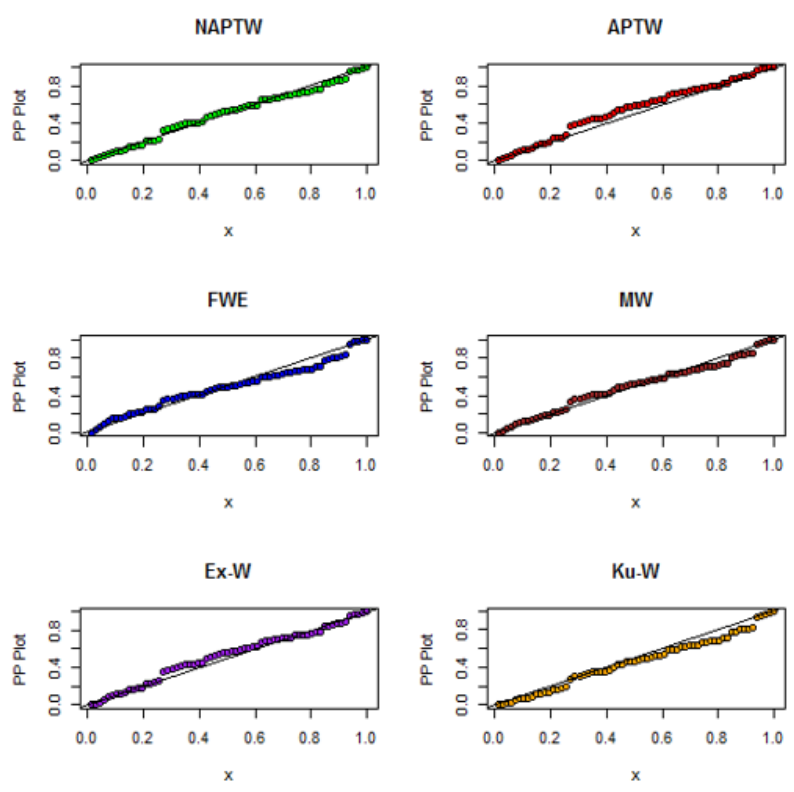

Figure 14: PP-plots of the fitted models using data 2.

\section{Concluding remarks}

In this article, a general class of distributions called new alpha power transformed family has been proposed. General expressions for the mathematical properties with estimation of parameters using three methods are discussed. For practical utility, we considered a three-parameter special model named as alpha power transformed Weibull distribution to evaluate the efficiency of the proposed class. The density function of the proposed model can take various forms such as negatively-skewed, positively skewed or symmetrical depending upon its parameters. Moreover, the hazard rate function of the suggested model can have monotonically increasing, decreasing, constant or bath-tub shaped hazard rates. For the illustrative purposes two examples are discussed and it is proved that the proposed method consistently provide better fit in modeling data than other distributions.

We hope that the new class and its special models will attract wider applications in numerous applied areas such as engineering, hydrology, survival, and lifetime data, economics, medical, among others. 


\section{References}

[1] Z. Ahmad, Z. Hussain, Flexible Weibull Extended Distribution, MAYFEB J. Materials Sci., 2 (2017), 5-18. 5

[2] A. M. Almarashi, M. Elgarhy, A new muth generated family of distributions with applications, J. Nonlinear Sci. Appl., 11 (2018), 1171-1184. 1

[3] M. Bourguignon, R. B. Silva, G. M. Cordeiro, The Weibull-G family of probability distributions, J. Data Sci., 12 (2014), 53-68. 1

[4] I. W. Burr, Cumulative frequency functions, Ann. Math. Statistics, 13 (1942), 215-232. 1

[5] G. M. Cordeiro, M. de Castro, A new family of generalized distributions, J. Stat. Comput. Simul., 81 (2011), $883-893$. 1

[6] G. M. Cordeiro, E. M. M. Ortega, S. Nadarajah, The Kumaraswamy Weibull distribution with application to failure data, J. Franklin Inst., 347 (2010), 1399-1429. 3

[7] S. Dey, V. K. Sharma, M. Mesfioui, A New Extension of Weibull Distribution with Application to Lifetime Data, Annals Data Sci., 4 (2017), 31-61. 1, 4

[8] M. Elgarhy, M. Haq, G. Ozel, N. Arslan, A new exponentiated extended family of distributions with Applications, Gazi University J. Sci., 30 (2017), 101-115. 1

[9] M. Elgarhy, A. S. Hassan, M. Rashed, Garhy-generated family of distributions with application, Math. Theory Model., 6 (2016), 1-15. 1

[10] N. Eugene, C. Lee, F. Famoye, The beta-normal distribution and its applications, Comm. Statist. Theory Methods, 31 (2002), 497-512. 1

[11] M. Haq, M. Elgarhy, The odd Frchet-G family of probability distributions, J. Stat. Appl. Prob., 7 (2018), 185-201. 1

[12] A. S. Hassan, M. Elgarhy, A New family of exponentiated Weibull-generated distributions, Int. J. Math. Appl., 4 (2016), 135-148. 1

[13] A. S. Hassan, M. Elgarhy, Kumaraswamy Weibull-generated family of distributions with applications, Adv. Appl. Stat., 48 (2016), 205-239. 1

[14] A. S. Hassan, M. Elgarhy, M. Shakil, Type II half Logistic family of distributions with applications, Pak. J. Stat. Oper. Res., 13 (2017), 245-264. 1

[15] S. Kotz, D. Vicari, Survey of developments in the theory of continuous skewed distributions, Metron, 63 (2005), $225-261$. 1

[16] C. D. Lai, M. Xie, D. N. P. Murthy, A modified Weibull distribution, IEEE Trans. Reliab., 52 (2003), 33-47. 2

[17] A. Mahdavi, D. Kundu, A new method for generating distributions with an application to exponential distribution, Comm. Statist. Theory Methods, 46 (2017), 6543-6557. 1, 1

[18] A. W. Marshall, I. Olkin, A new method for adding a parameter to a family of distributions with application to the exponential and Weibull families, Biometrika, 84 (1997), 641-652. 1

[19] G. S. Mudholkar, D. K. Srivastava, Exponentiated Weibull family for analyzing bathtub failure rate data, IEEE Trans. Reliab., 42 (1993), 299-302. 1, 1

[20] S. Nadarajah, S. Kotz, Strength modeling using Weibull distributions, J. Mech. Sci. Tech., 22 (2008), 1247-1254. 8

[21] G. P. Patil, J. K. Ord, On size-biased sampling and related form-invariant weighted distributions, Sankhya, Ser. B, 38 (1976), 48-61. 1

[22] G. P. Patil, C. R. Rao, The weighted distributions: a survey of their applications. In Applications of Statistics, P. R. Krishnaiah (ed.), 383-405, North Holland Publishing Company, Amesterdam, (1977). 1

[23] K. Pearson, Contributions to the mathematical theory of evolution. II. Skew variation in homogeneous material, Philos. Trans. R. Soc. Lond. A, 186 (1895), 343-414. 1

[24] A. Saboor, T. K. Pogány, Marshall-Olkin gamma-Weibull distribution with applications, Comm. Statist. Theory Methods, 45 (2016), 1550-1563. 8 